\title{
Anti-disturbance Attitude Control of Flexible Satellite Based on T-S Fuzzy Modeling
}

\author{
Bin Hang*, Songyin Cao \\ College of Information Engineering, Yangzhou University, Yangzhou, 225000, China. \\ * Corresponding author. Email: 350754315@qq.com \\ Manuscript submitted January 10, 2019; accepted March 8, 2019. \\ doi: 10.17706/ijcee.2019.11.2.91-100
}

\begin{abstract}
In this paper, in view of the multiple disturbances, the problem of anti-disturbance attitude control of the microsatellite in orbit is studied. The T-S fuzzy model is introduced to identify the external disturbance, and the fuzzy disturbance observer is designed to observe and compensate the unknown disturbance, especially for some irregular external disturbance. Based on the Lyapunov function method, the controller gain and the disturbance observer gain are calculated to ensure the stability of the satellite attitude control system. It can be concluded from the simulation results that the proposed anti-disturbance attitude control method based on T-S fuzzy modeling can improve the accuracy and stability of the attitude control system.
\end{abstract}

Keywords: Attitude control, disturbance observer, microsatellite, T-S fuzzy.

\section{Introduction}

Since the Soviet Union launched the world's first artificial satellite in 1957, the prelude to human space exploration has been revealed [1]. The satellite attitude control system (ACS) is an important part of the satellite system. With the rapid development of modern aerospace technology, the scientific research tasks required by satellites have become more and more diverse, and the performance requirements of satellite attitude control systems are becoming higher and higher. In [2], the frequency domain disturbance observer was designed for disturbance estimation and compensation. A composite controller combining the frequency domain disturbance observer with PID (Proportional Integral Derivative) controller is presented to reject and attenuate the multiple disturbances. In [3], an anti-disturbance fault tolerance control (FTC) method based on output feedback control was proposed for satellite attitude control system with partially measurable state and the reaction wheel fault. An anti-saturation FTC method based on disturbance observer is proposed for satellites with actuator failure, input saturation and multiple disturbance in [4]. In [5]-[9], sliding mode control methods based on disturbance observer were proposed for the microsatellite attitude control system with multiple disturbances and flywheel fault. Flexible satellites usually refer to structures consisting of a central rigid body and flexible attachments. Many modern spacecraft have large, complex, lightweight and flexible attachments. Fig. 1 is a schematic diagram of the attitude control system of the flexible satellite.

Therefore, the performance of attitude control system is inevitably affected by the vibration of flexible accessories. In the past decades, research on attitude control of flexible satellites has been a hot topic in the field of control. The most classic control method among these control methods is PID control [10]. In [11], a 
robust adaptive FTC method for attitude tracking was designed for the flexible spacecraft with reaction flywheel fault, external disturbance and unknown inertia parameter uncertainty. High-precision attitude control and vibration suppression are successfully achieved when considering various situations of failure. In order to improve the performance of sliding mode control, a neural network-based adaptive controller was applied to compensate the modeling errors [12]. Reference [13] considered the attitude control problem of a flexible spacecraft with time-varying control input delay. A composite disturbance observer based control (DOBC) and $H_{\infty}$ control scheme was applied to control the spacecraft attitude, and improve the stability effectively.

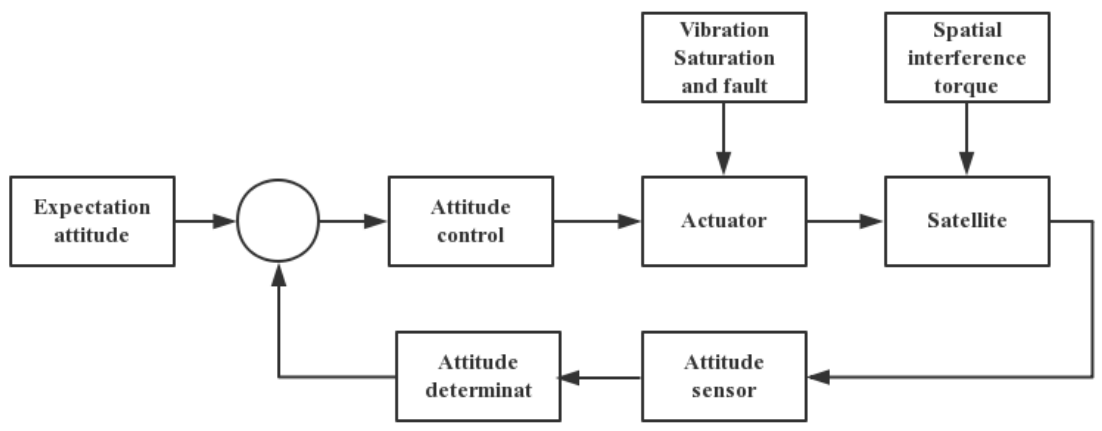

Fig. 1. Flexible satellite attitude control system diagram.

In order to improve the anti-disturbance ability of the system, the engineering community proposed the DOBC method in the 1980s, which is a control method based on the disturbance observer. The basic idea is to use the observer to estimate the external disturbance and apply it in the feedforward channel [14]. However, in the existing DOBC literature, the modeling disturbance of external system often considers the constant disturbance or harmonic disturbance generated by a linear system. For those nonlinear or irregular disturbance, the existing DOBC disturbance modeling method is no longer applicable, and it is urgent to find a new modeling method.

In this paper, we introduce the T-S fuzzy model to identify the external disturbance, and combine the fuzzy disturbance observer to observe and compensate the unknown disturbance, especially for some irregular external disturbance. Furthermore, the composite controller is designed in combination with the disturbance observer and the PD control input to ensure that the system still has good stability under the disturbance of external disturbance.

\section{Flexible Satellite Dynamics Model}

The structure of a flexible satellite generally consists of a flexible attachment and a rigid body structure. Due to the complexity of the derivation process, this paper uses the Lagrange function as an aid to derive the attitude dynamics model of a flexible satellite [15], [16]. It is possible to directly write the three-axis flexible satellite attitude dynamics equation as:

$$
\begin{aligned}
& \boldsymbol{J} \ddot{\theta}+F \ddot{\boldsymbol{\eta}}=u(\mathrm{t})+\mathrm{d}_{1}(t) \\
& \ddot{\boldsymbol{\eta}}+2 \varepsilon \rho \dot{\eta}+\rho^{2} \eta+F^{T} \ddot{\theta}(t)=0
\end{aligned}
$$

where $J$ are three-axis inertia moments, $\theta$ is the attitude angle, $F$ is the coupling matrix of the rigid body and the flexible attachment; $u(t), \eta(t)$ represent the control input and modal. The damping matrix is 


$$
2 \varepsilon \rho=\operatorname{diag}\left\{2 \varepsilon_{i} \rho_{i}, i=1,2 \ldots n\right\}
$$

where $\varepsilon_{i}(i=1,2, \ldots . n)$ is the damping ratio, and $\rho_{i}(i=1,2, \ldots n)$ is the modal frequency; $d_{1}(t)$ is the space environment disturbance matrix, and its value is equivalent. Dynamic systems (1) and (2) can be converted to the following equation after a series of algebraic operations:

$$
\left(J-F F^{T}\right) \ddot{\theta}=u(t)+d_{0}(t)+d_{1}(t)
$$

where the vibration generated by the flexible attachment can be considered as

$$
d_{0}(t)=F\left(2 \varepsilon \rho \dot{\eta}+\rho^{2} \eta\right)
$$

Denote $x(t)=[\theta, \dot{\theta}]^{T}$, then the following equations can be acquired as:

$$
\dot{x}(t)=A x(t)+B_{0}\left[u(t)+d_{0}(t)\right]+B_{1} d_{1}(t)
$$

where

$$
A=\left[\begin{array}{ll}
0 & 1 \\
0 & 0
\end{array}\right] \quad B_{0}=B_{1}=\left[\begin{array}{c}
0 \\
\left(J-F F^{T}\right)^{-1}
\end{array}\right]
$$

\section{Anti-disturbance Composite Controller Design}

The previous section mainly introduces the establishment of flexible satellite dynamics model and introduces the common methods of satellite attitude control. This section will introduce the design of T-S fuzzy disturbance modeling and anti-disturbance composite control.

The famous T-S fuzzy system was proposed by Sugeno and Takagi in 1985. By decomposing the whole state space into multiple fuzzy subspaces to describe complex linear systems, each subspace can be transformed by simple linear regression. The model shows that such a fuzzy system can be used to approximate any irregular, nonlinear disturbance system.

On the one hand, the T-S fuzzy system can well describe a nonlinear systems. Because the latter part of the disturbance rule takes the form of linear or nonlinear functions of the input signal, each rule contains more information, so fewer rules can be applied to express more system information. In recent years, experts and scholars have done a lot of research on T-S models, which are widely used in fuzzy system modeling. On the other hand, T-S fuzzy models has been widely used to identify or approximate irregular complex nonlinear systems. By introducing some fuzzy rules, the T-S fuzzy model can approximate many nonlinear control systems and even accurately describe a specific type of nonlinear dynamics. It can be seen that many irregular and nonlinear complex disturbances can be combined with the T-S fuzzy system modeling method. The disturbance observer is further combined with the PD control algorithm to ensure that the system can still ensure the stability of the system under the following conditions.

\subsection{Design of T-S Fuzzy Observer}

According to the disturbance observer designed in [17], constant values and harmonic disturbance can be observed. It is worth noting that certain types of disturbance in actual control systems can often be generated by using linear external systems, such as unknown constants and harmonic signals,. It is difficult to describe those irregular external disturbances based on linear exogenous systems. Therefore, a disturbance observer is designed as a whole T-S disturbance model, as shown in (5) below: 


$$
\left\{\begin{array}{l}
\dot{\omega}(t)=\sum_{j=1}^{r} h_{j}(\theta) \omega(t) \\
d_{0}(t)=\sum_{j=1}^{r} h_{j}(\theta) V_{j} \omega(t)
\end{array}\right.
$$

In order to better estimate the disturbance model $d_{0}(t)$ in (5), the disturbance observer is designed as follows.

$$
\left\{\begin{array}{l}
\hat{d}_{0}(t)=\sum_{j=1}^{r} h_{j}(\theta) V_{j} \omega(t) \\
\hat{\omega}(t)=\varepsilon(t)-L x(t) \\
\dot{\varepsilon}(t)=\sum_{j=1}^{r} h_{j}(\theta)\left\{\begin{array}{l}
{\left[\left(W_{j}+L B_{0} V_{j}\right)(\varepsilon(t)-L x(t))\right]} \\
+L\left[A x(t)+B_{0} u(t)\right]
\end{array}\right\}
\end{array}\right.
$$

where $\hat{\omega}(t)$ is the estimation of $\omega(t), \hat{d}_{0}(t)$ is the estimation of $d_{0}(t), L$ is the gain matrix to be determined later and $\varepsilon(t)$ is an auxiliary variable.

\subsection{Design of Composite Anti-disturbance Controller Design}

For the unbounded energy bounded disturbance in the system, firstly, the state feedback controller is constructed as follows:

$$
u_{f}(t)=M x(t)
$$

where $M$ is the state feedback controller gain to be determined later. Therefore, an anti-disturbance composite controller based on the PD controller and the disturbance observer can be constructed:

$$
u(t)=u_{f}(t)-\hat{d}_{0}(t)=M x(t)-\hat{d}_{0}(t)
$$

Bringing equation (8) into system (4) can be expressed as:

$$
\dot{x}(t)=\sum_{j=1}^{r} h_{j}(\theta)\left[\begin{array}{l}
\left(A+B_{0} M\right) x(t)+ \\
B_{0} V_{j} e_{w}(t)+B_{1} d_{1}(t)
\end{array}\right]
$$

Define disturbance estimation error

$$
e_{w}(t)=\omega(t)-\hat{\omega}(t)
$$

From equations (4-6), we can get the estimation error equations:

$$
\begin{aligned}
\dot{e}_{w}(t)= & \sum_{j=1}^{r} h_{j}(\theta) W_{j} \omega(t)-\dot{\varepsilon}(t)+L \dot{x}(t) \\
= & \sum_{j=1}^{r} h_{j}(\theta) W_{j} \omega(t)-\sum_{j=1}^{r} h_{j}(\theta)\left\{\begin{array}{l}
{\left[\left(W_{j}+L B_{0} V_{j}\right)(\varepsilon(t)-L x(t))\right]} \\
+L\left[A x(t)+B_{0} u(t)\right]
\end{array}\right\} \\
& +\sum_{j=1}^{r} h_{j}(\theta)\left\{\begin{array}{l}
L A x(t)+L B_{0} M x(t)+L B_{0} M x(t) \\
+L B_{0} V_{j} e_{w}(t)+L B_{1} d_{1}(t)
\end{array}\right\} \\
= & \sum_{j=1}^{r} h_{j}(\theta)\left[\left(W_{j}+L B_{0} V_{j}\right) e_{w}(t)+L B_{1} d_{1}(t)\right]
\end{aligned}
$$


Therefore, the closed-loop system available from equations (11) and (12):

$$
\left[\begin{array}{l}
\dot{x}(t) \\
\dot{e}_{w}(t)
\end{array}\right]=\sum_{j=1}^{r} h_{j}(\theta)\left\{\begin{array}{cc}
{\left[\begin{array}{cc}
A+B_{0} M & B_{0} V_{j} \\
0 & W_{j}+L B_{0} V_{j}
\end{array}\right]\left[\begin{array}{c}
x(t) \\
e_{w}(t)
\end{array}\right]} \\
+\left[\begin{array}{c}
B_{1}(t) \\
L B_{1}(t)
\end{array}\right] d_{1}(t)
\end{array}\right\}
$$

Combining (10) with (11), the concerned system can be formulated by

$$
\left\{\begin{array}{l}
\dot{e}(t)=\left(W_{1}+N B_{0}\right) e(t)+T d_{1}(t) \\
Z_{\infty}(t)=C e(t)
\end{array}\right.
$$

where $Z_{\infty}(t)$ is the $H_{\infty}(t)$ reference output, $e(t)=\left[\begin{array}{c}x(t) \\ e_{w}(t)\end{array}\right], T=\left[\begin{array}{c}I \\ L\end{array}\right], N=\left[\begin{array}{cc}M & V_{j} \\ 0 & L V_{j}\end{array}\right], W_{1}=\left[\begin{array}{cc}A & 0 \\ 0 & W_{j}\end{array}\right]$,

$C$ is the $H_{\infty}$ reference output matrix.

The designed approach of the anti-disturbance control can be further formulated as follows.

Theorem 1: For $\gamma_{1}>0$, if there exit matrices $P>0, R$ satisfying

$$
\Theta_{1}=\left[\begin{array}{ccc}
\operatorname{sym}\left(P W_{1}+R B_{0}\right) & P T & C_{1}^{T} \\
* & -\gamma_{1}^{2} & 0 \\
* & * & -I
\end{array}\right]<0
$$

Then with gain $\left[\begin{array}{c}L \\ M\end{array}\right]=P^{-1} R$, the estimation error system (11) is stable and satisfies

$$
\left\|Z_{\infty}(t)\right\|_{2}^{2}<\gamma_{1}^{2}\left\|d_{1}(t)\right\|_{2}^{2}
$$

Proof: Define a Lyapunov function

$$
V_{1}(t)=e^{T} P e
$$

For the constant $\gamma_{1}>0$, the $H_{\infty}$ performance index is defined as

$$
J_{\infty}(t)=\int_{0}^{\infty}\left(Z_{\infty}^{T}(t) Z_{\infty}(t)-\gamma_{1}^{2} d_{1}^{T}(t) d_{1}(t)+\dot{\gamma}_{1}(t)\right) d(t)+V_{1}(t)
$$

The derivation of auxiliary function is

$$
\begin{aligned}
\dot{J}_{\infty}(t)= & Z_{\infty}^{T}(t) Z_{\infty}(t)-\gamma_{1}^{2} d_{1}^{T}(t) d_{1}(t)+ \\
& \dot{e}^{T}(t) P e(t)+e^{T}(t) P \dot{e}(t) \\
= & {\left[\begin{array}{c}
e(t) \\
d_{1}(t)
\end{array}\right]\left[\begin{array}{cc}
\Omega_{11} & P T \\
* & -\gamma_{1}^{2} I
\end{array}\right]\left[\begin{array}{l}
e(t) \\
d_{1}(t)
\end{array}\right] }
\end{aligned}
$$

where 


$$
R=P N, \Omega_{11}=\operatorname{sym}\left(P W_{1}+R B_{0}\right)+C_{1}^{T} C_{1}
$$

Define

$$
\Omega_{1}=\left[\begin{array}{cc}
\Omega_{11} & P T \\
* & -\gamma_{1}^{2} I
\end{array}\right]
$$

If $\Omega_{1}$ is left and right multiplied by $\operatorname{diag}\left\{P^{-1}, I\right\}$,it can be seen that $J_{\infty}<0$. Furthermore, we have $\dot{V}_{1}(t)<0,\left\|Z_{\infty}(t)\right\|_{2}^{2}<\gamma_{1}^{2}\left\|d_{1}(t)\right\|_{2}^{2}$ and $J_{\infty}(t)=\int_{0}^{\infty}\left(Z_{\infty}^{T}(t) Z_{\infty}(t)-\gamma_{1}^{2} d_{1}^{T}(t) d_{1}(t)+\dot{\gamma}_{1}(t)\right) d(t)+V_{1}(t)$. The proof is completed.

\section{Simulation Examples}

Assume that the flexible microsatellite operating in a circular orbit with the altitude of $900 \mathrm{~km}$ given in [18], [19]. The orbital angular velocity is $n_{0}=0.0011 \mathrm{rad} / \mathrm{s}$. The parameters of the spacecraft are obtained from [20], [21], three-axis inertia moment are

$$
J=\left[\begin{array}{ccc}
5.5 & 0 & 0 \\
0 & 6.14 & 0 \\
0 & 0 & 2.18
\end{array}\right]
$$

Similar to [20], only the first two elastic modes are used to describe the vibration of the flexible appendage with

$$
\omega_{1}=3.17, \omega_{2}=7.38
$$

The corresponding damping is

$$
\xi_{1}=0.001, \xi_{2}=0.0015
$$

The rigid-elastic coupling matrix is adopted as

$$
F=\left[\begin{array}{ll}
1.2781 & 0.9176
\end{array}\right]
$$

The space environment disturbance is supposed to be

$$
d_{1}(t)=4.5 \times 10^{-5}\left(3 \cos \omega_{0} t+1.5 \sin \omega_{0} t\right) N m .
$$

Taking the pitch angle of a satellite as an example. The initial angular velocity is $\dot{\theta}(0)=0.001 \mathrm{rad} / \mathrm{s}$ and the initial attitude is $\theta(0)=0.05 \mathrm{rad}$.

Selecting the following Gaussian function as the membership function of the T-S fuzzy system:

$$
A_{1}^{1}=\frac{\exp \left(\frac{-\left(z_{1}-1.5\right)^{2}}{2 \sigma_{1}^{2}}\right)}{\exp \left(\frac{-\left(z_{1}-1.5\right)^{2}}{2 \sigma_{1}^{2}}\right)+\exp \left(\frac{-\left(z_{1}-1\right)^{2}}{2 \sigma_{2}^{2}}\right)}
$$




$$
A_{1}^{2}=\frac{\exp \left(\frac{-\left(z_{1}-1\right)^{2}}{2 \sigma_{2}^{2}}\right)}{\exp \left(\frac{-\left(z_{1}-1.5\right)^{2}}{2 \sigma_{1}^{2}}\right)+\exp \left(\frac{-\left(z_{1}-1\right)^{2}}{2 \sigma_{2}^{2}}\right)}
$$

For the disturbance system (5), nonlinear disturbance can be described as

$$
\begin{gathered}
W_{1}=\left[\begin{array}{ll}
-1 & 2 \\
-5 & 0
\end{array}\right], W_{2}=\left[\begin{array}{cc}
0 & -6 \\
4 & 0
\end{array}\right], \\
V_{1}=\left[\begin{array}{l}
0 \\
4
\end{array}\right], V_{2}=\left[\begin{array}{l}
0 \\
3
\end{array}\right],
\end{gathered}
$$

where

$$
\sigma_{1}^{2}=0.5, \sigma_{2}^{2}=1
$$

For $H_{\infty}$ attenuation indices $\gamma_{1}=1.55$, from theorem 1,we can see that the gains of disturbance observer and PD controller are

$$
\begin{gathered}
M=\left[\begin{array}{ll}
-1.4221 & -1.9368
\end{array}\right] \\
L=\left[\begin{array}{cc}
12.6599 & -12.0191 \\
-8.0663 & 8.0245
\end{array}\right]
\end{gathered}
$$

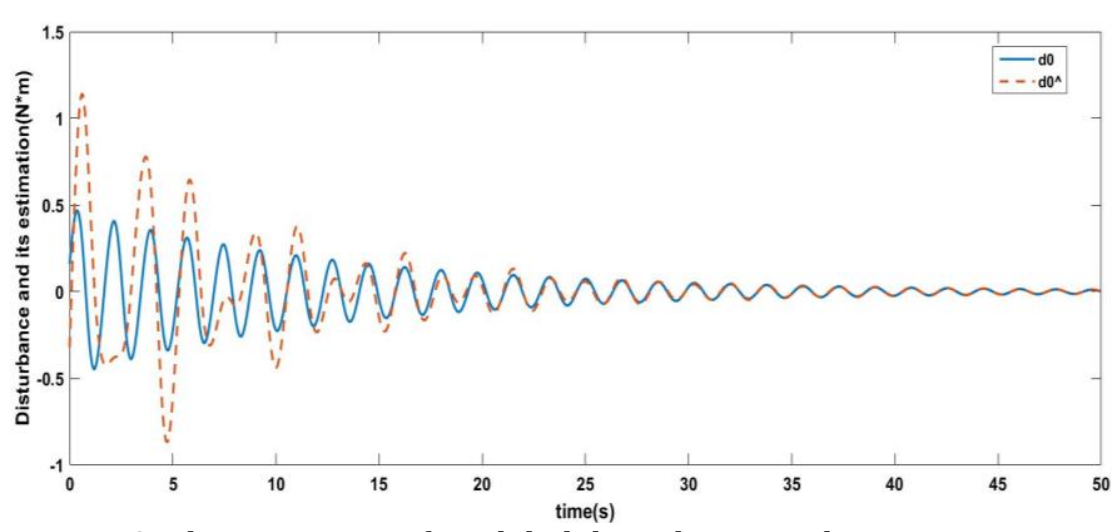

Fig. 2. The responses of modeled disturbance and its estimation.

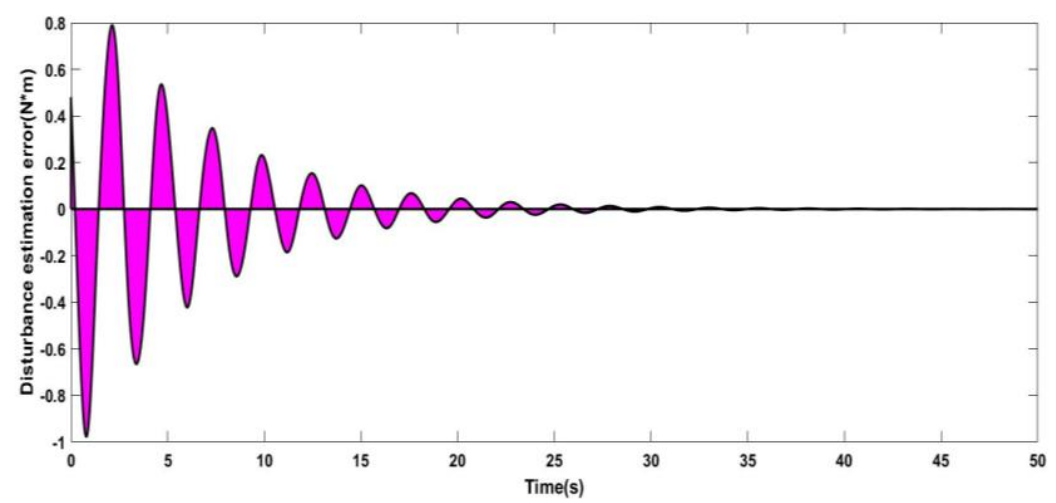

Fig. 3. The estimation error of modeled disturbance. 
Fig. 2 demonstrates the responses of modeled disturbance $d(t)$ and its estimation. Fig. 3 shows the estimation error of modeled disturbance. From Figs. 2 and Fig. 3, the disturbance observer has a good estimation accuracy for a flexible satellite, it easy to know the T-S fuzzy disturbance observer can estimate the disturbance.

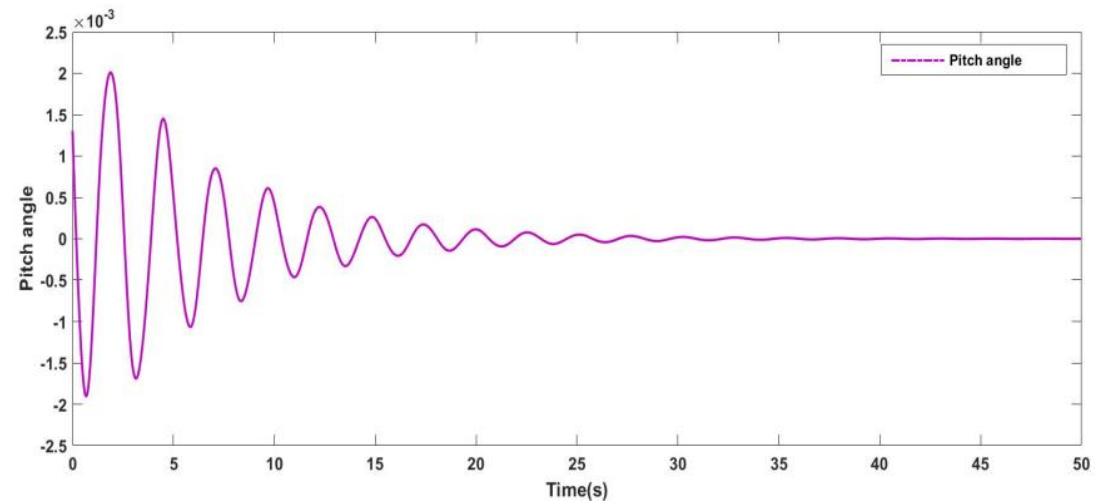

Fig. 4. The responses of pitch angle.

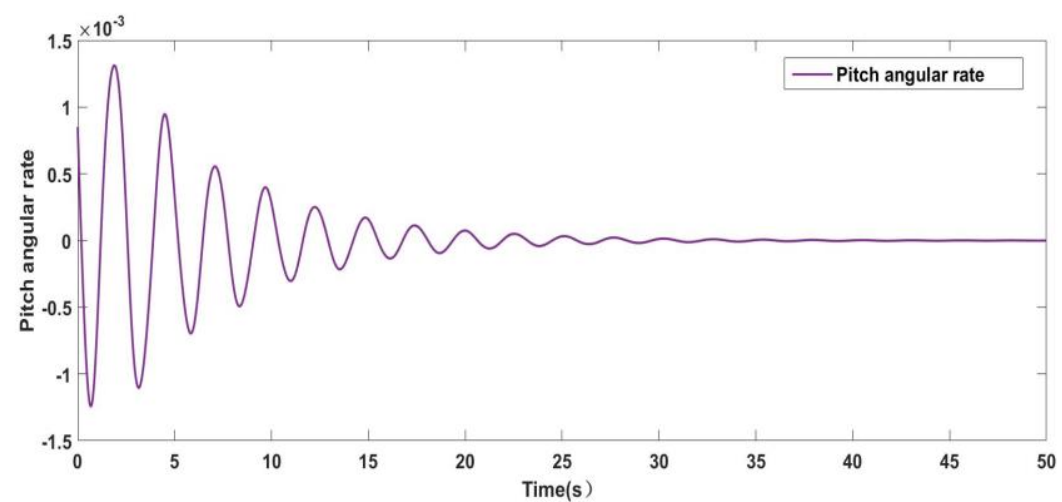

Fig. 5. The responses of pitch attitude angle rate.

Fig. 4 and Fig. 5 show the pitch attitude angle and pitch attitude angle rate. From Fig. 4 and Fig. 5, it can be seen that the proposed anti-disturbance composite control has good anti-disturbance ability.

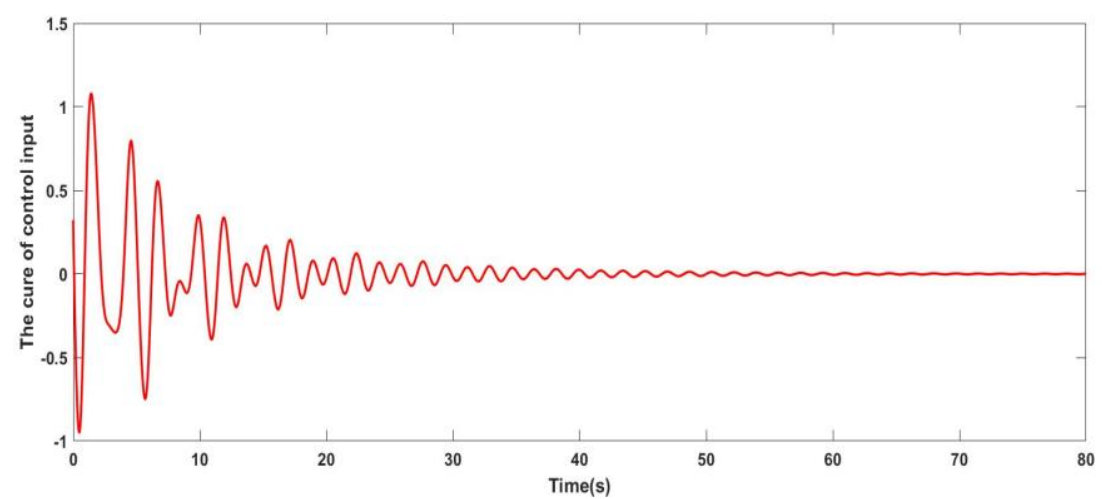

Fig. 6. The responses of control input.

In Fig. 6, we can see the control input curve of the system and it can be seen that the system has good stability. 


\section{Conclusion}

In this paper, the external irregular disturbance is modeled by T-S fuzzy model. For the attitude control problem of flexible satellites with multiple disturbances, an attitude control method based on T-S fuzzy disturbance modeling is proposed. Based on the T-S model disturbance modeling, and the PD control combined with the disturbance observer is designed to design the composite controller. Different from the previous disturbance observer, T-S fuzzy modeling technology is applied to describe the elastic modal. Therefore, the system still has good stability under the influence of external disturbance. Finally, the effectiveness of the proposed method is verified by simulation of the microsatellite attitude control system.

\section{Acknowledgment}

This work is partially supported by the National Nature Science Foundation of China (Grant no.61873346).

\section{References}

[1] Chen, M., Ren, B. B., Wu, Q. X., et al. (2015). Anti-disturbance control of hypersonic flight vehicles with input saturation using disturbance observer. Journal of Science China Information Sciences, 58(7), 1-12.

[2] Chi, W. J., Qiao, J. Z., Zhu, Y. K., et al. (2015). Disturbance estimation and compensation for drag-free satellite based on frequency domain disturbance observer. Proceedings of the 54th Annual Conference of the Society of Instrument and Control Engineers of Japan (SICE) (pp. 284-300).

[3] Hu, Q. L., Jiang, B. Y., Shi, Z., et al. (2014). Novel terminal sliding mode based fault tolerant attitude control for spacecraft under actuator faults. Journal of Acta Aeronautica et Astronautica Sinica, 35(1), 249-258.

[4] Cao, S., \& Zhao, Y. (2017). Anti-disturbance fault-tolerant attitude control for satellites subject to multiple disturbances and actuator saturation. Journal of Nonlinear Dynamics, 89(6), 1-11.

[5] Chen, W. H. (2005). Disturbance observer based control for nonlinear systems. Journal of the Transactions on Mechatronics, 9(4), 706-710.

[6] Yang, J., Li, S. H., \& Yu, X. H. (2013). Sliding-mode control for systems with mismatched uncertainties via disturbance observer. Journal of Transactions on Industrial Electronics, 60(1), 160-169.

[7] Xiao, B., Hu, Q. L., \& Zhang, Y. (2011). Adaptive sliding mode fault tolerant attitude tracking control for flexible spacecraft under the actuator saturation. Journal of the Control Technology, 20(6), 1605-1612.

[8] Guo, L., \& Cao, S. (2014). Anti-disturbance control theory for systems with multiple disturbances. ISA Transactions, 53(4), 846-849.

[9] Zhang, R., Li, T., \& Guo, L. (2013). Disturbance observer based, control for flexible spacecraft with timevarying input delay. Journal of the Advances in Difference Equations, 2013(1), 1-12.

[10] Ma, Y. J., Jiang, B., Gang, T., et al. (2013). Actuator failure compensation and attitude control for rigid satellite by adaptive control using quaternion feedback. Journal of the Franklin Institute, 351(1), 296314.

[11] Jiang, Y., Hu, Q. L., \& Ma, G. (2009). Adaptive backstepping fault-tolerant control for flexible spacecraft with unknown bounded disturbances and actuator failures. ISA Transactions, 49(1), 57-69.

[12] Bae, J., \& Kim, Y. (2012). Adaptive controller design for spacecraft formation flying using sliding mode controller and neural networks. Journal of the Franklin Institute, 349(2), 578-603.

[13] Zhang, B., Li, T., \& Zheng, B. C. (2016). Control of flexible spacecraft with disturbance and delay. Journal of the Information \&Control, 45(4), 407-414.

[14] Liu, H., Guo, L., \& Zhang, Y. M. (2012). An anti-disturbance pd control scheme for attitude control and stabilization of flexible spacecrafts. Journal of the Nonlinear Dynamic, 67(3), 2081-2088. 
[15] Singhose, W. E., Banerjee, A. K., \& Seering, W. P. (2014). Slewing flexible spacecraft with deflectionlimiting input shaping. Journal of Guidance, Control, and Dynamics, 35(1), 249-258.

[16] Hu, Q. L., \& Ma, G. (2005). Variable structure control and active vibration suppression of flexible spacecraft during attitude maneuver. Journal of the Aerospaceence \& Technology, 9(4), 307-317.

[17] Yang, J., Li, S. H., \& Yu, X. H. (2013). Sliding-mode control for systems with mismatched uncertainties via a disturbance observer. Journal of Industrial Electronics, 60(1), 160-169.

[18] Liu, Z. J., Liu, J. K., \& Wang, L. J. (2018). Disturbance observer based attitude control for flexible spacecraft with input magnitude and rate constraints. Journal of the Aerospace Science \&Technology, 72(1), 486-492.

[19] Chen, M., Shao, S. Y., \& Jiang, B. (2017). Adaptive neural control of uncertain nonlinear systems using disturbance observer. IEEE Transactions on Cybernetics, 99, 1-14.

[20] Cao, S., Yi, Y., \& Guo, L. (2014). Anti-disturbance fault diagnosis for non-Gaussian stochastic distribution systems with multiple disturbances. Journal of the Neurocomputing, 136, 315-320.

[21] Cao, S., Guo, L., \& Chen, W. (2017). Anti-disturbance fault tolerant initial alignment for inertial navigation system subjected to multiple disturbances. Journal of the Aerospace Science and Technology, 72, 95-103.

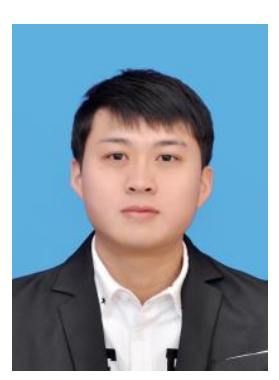

Bin Hang received the B.Sc. degree from the College of Information Engineering, Southeast University Chengxian College, Nanjing, China, in 2017. Now, he is a master candidate student at Yangzhou University. His current research interests include antidisturbance control and fault tolerant control.

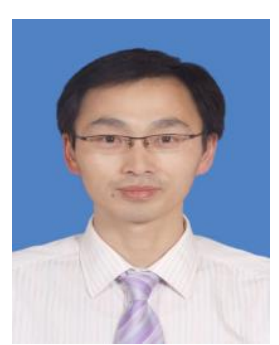

Songyin Cao received his M.S. degree from College of Information Engineering, Yangzhou University, Yangzhou, China, in 2006, and Ph.D. degree from School of Automation, Southeast University, Nanjing, China, in 2011. From 2012 to 2015, he was a post-doctoral fellow at Beihang University.

From 2014 to 2015, he was an academic visitor at Loughborough University, UK. Now, he is an associate professor at Yangzhou University. His current research interests include fault tolerant control and inertial navigation system. 